\title{
Erratum for "Fluctuating Asymmetry and Growth as Biomarkers for Exposure to Androgen Disrupting Chemicals in Japanese Quail" [10.1007/s10646-005-0013-9(10646-2005-13 Quinn)]
}

Published online 1 October 2005

\author{
Author name is not correct in abstract online. \\ Abstract online reads: MICHAEL J. C.L. SUMMITT, K. BURRELL, M.A. OTTINGER \\ Correct names are: \\ MICHAEL J. QUINN JR., C.L. SUMMITT, K. BURRELL, M.A. OTTINGER \\ (Online pdf is correct)
}

This was a typesetter's error. 